\title{
Développement de Dipetalonema (A.) weissi, Filaire de Macroscélide, chez un Ornithodore
}

\author{
par O. BAIN et J.-C. QUENTIN * \\ Laboratoire de Zoologie (Vers) associé au C.N.R.S., \\ Muséum national d'Histoire naturelle, 43, rue Cuvier, F 75231 Paris Cedex 05, \\ et Laboratoire de Biologie, Université des Sciences et Techniques, \\ Sfax, République Tunisienne.
}

\section{Résumée.}

Les microfilaires de $D$. (A.) weissi, parasite d'Elephantulus rozeti en Afrique du Nord, évoluent jusqu'au stade infestant chez Ornithodoros erraticus (souche Vellozo de Casablanca).

La Filaire $D$. (A.) viteae, parasite des Rongeurs Gerbillidae, est très proche de $D$. (A.) w'eissi, mais s'en distingue aisément; les stades infestants, au contraire, nous paraisesnt indistinguables.

\section{Summary.}

Development of D. (A.) veissi, a Macroscelid's Filaria, in Ornithodoros erraticus.

The microfilariae of D. (A.) weissi parasite of Elephantulus rozeti in North Africa evolved in Ornithodoros erraticus (Vellozo strain from Casablanca) where they reach the infective stage.

The Filaria $D$. (A.) viteae, parasite of Gerbillid Rodents, is closely related to $D$. (A.) weissi but has some clear differential characters; on the contrary the infective stages seem to be undistinguishable.

* Ce travail a été effectué grâce à une subvention de l'Organisation Mondiale de la Santé.

Reçu le 10 avril 1977. 


\section{Introduction}

La Filaire Dipetalonema (Acanthocheilonema) weissi (Seurat, 1914), parasite d'Elephantulus rozeti (Duvernoy, 1833), décrite initialement en Algérie, a été retrouvée aux environs de Sfax, en Tunisie. Un de ces Elephantulus parasité, a été apporté vivant à Paris pour tenter de réaliser le cycle larvaire, encore inconnu.

D. (A.) weissi étant morphologiquement très proche de D. (A.) viteae (Krepkogorskaya, 1933), dont le cycle s'effectue chez un Ornithodore (Balthazard et coll., 1952), des Ornithodoros erraticus (souche Vellozo), maintenus en élevage, ont été gorgés sur l'Elephantulus. A $27^{\circ} \mathrm{C}$, des stades infestants ont été obtenus 24 jours plus tard.

\section{Complément à l'étude des adultes}

L'Elephantulus est mort après le gorgement des Ornithodores. A l'autopsie, 7 et $4 \delta^{\top}$ ont été récoltés: $1 \uparrow$ dans le péritoine, les autres spécimens dans le tissu sous-cutané, au niveau de la tête, du ventre et du dos.

Femelles et mâles ont une tête arrondie, des déirides bien marquées, situées au niveau du début de l'œsophage, un œsophage très nettement divisé, et une queue longue portant 2 grandes languettes sub-terminales.

Les mensurations sont les suivantes: pour une des femelles, corps long de $2,9 \mathrm{~cm}$ et large de $250 \mu$; anneau nerveux et déirides à 260 et $500 \mu$ de l'apex; œsophage long de $3000 \mu$ avec portion musculaire de $530 \mu$; vulve à $910 \mu$ de l'apex et portion impaire de l'ovéjecteur longue de $1100 \mu$; queue longue de $330 \mu$; microfilaire utérine longue de $195 \mu$. Les corps de 2 autres femelles sont longs de 3,3 et $3,2 \mathrm{~cm}$, avec des œsophages longs respectivement de 3200 et $3550 \mu$. Un des mâles est long de $2 \mathrm{~cm}$ et large de $145 \mu$, avec un œsophage long de $2580 \mu$; la queue est longue de $280 \mu$, les spicules gauche et droit longs de 370 et $150 \mu$. Le corps et l'œsophage sont mesurés également sur un autre mâle: ils sont longs respectivement de $1,86 \mathrm{~cm}$ et $2870 \mu$.

Par leurs dimensions et leur morphologie (fig. 1), nos spécimens correspondent bien à $D$. (A.) weissi (Seurat, 1914). Notre matériel permet d'ajouter quelques précisions morphologiques à l'excellente description de Seurat:

— La tête porte un cercle de 6 papilles labiales externes dont 2 sont accolées aux amphides (papilles dorso-latérales) et 4 papilles céphaliques, disposées en carré.

- L'area rugosa est présente; elle commence à $650 \mu$ de l'extrémité caudale et s'étend sur $3400 \mu$; elle est constituée de bandes transversales de petits bâtonnets; l'écartement des bandes est d'une dizaine de $\mu$.

- Le spicule droit porte des sillons transversaux dans la région sub-apicale; le spicule gauche a une lame de forme simple, terminée par une pointe conique. 


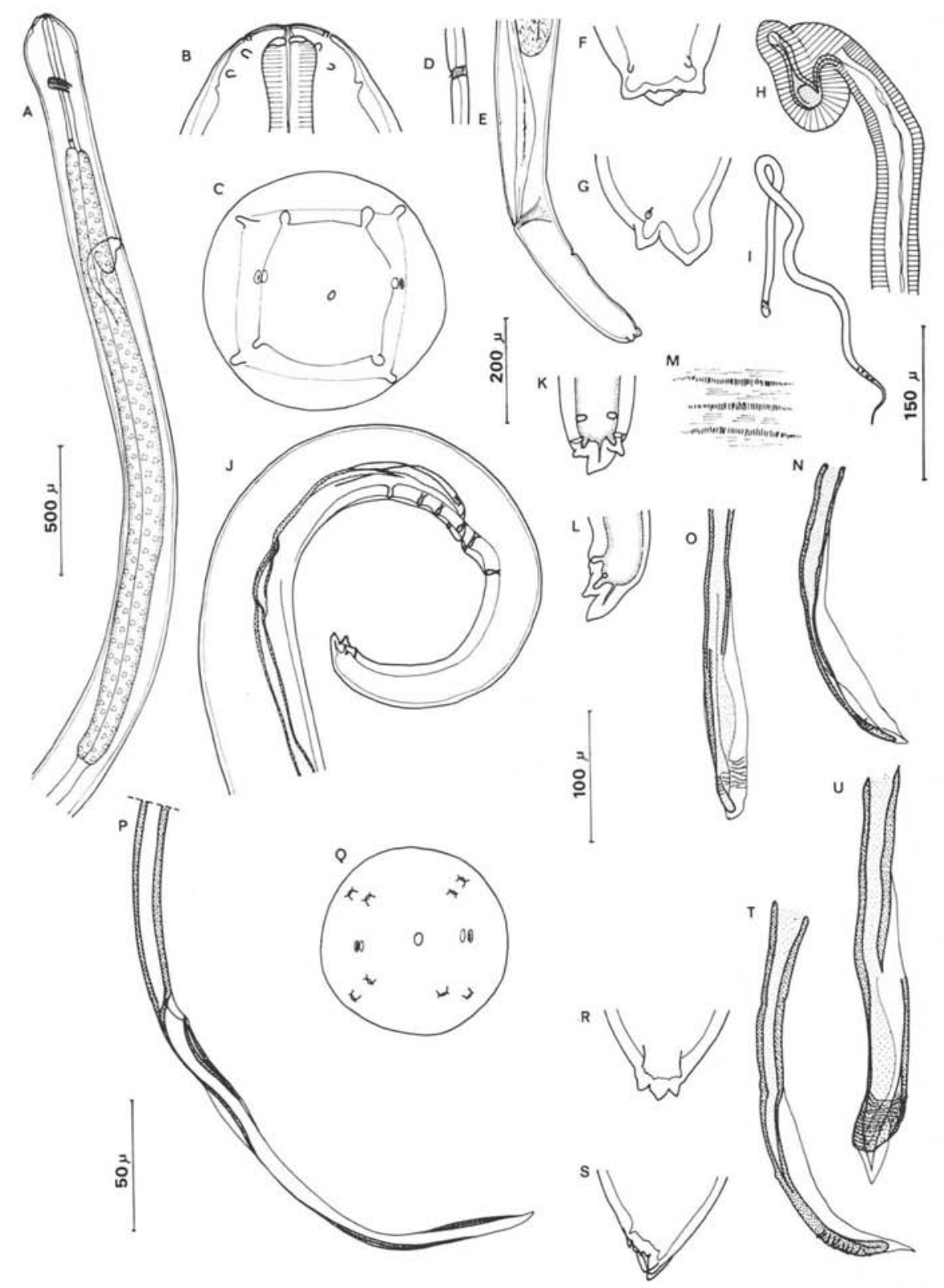

Fig. 1. - $\mathrm{A}$ à $\mathrm{P}, \mathrm{D}$. (A.) weissi. $\mathrm{A}$ à $\mathrm{I}$, femelle; $\mathrm{A}$ : région antérieure, vue latérale; $\mathrm{B}$ et $\mathrm{C}$ : tête en vues médiane et apicale; $\mathrm{D}$ : déiride vue de profil ; $\mathrm{E}$ : région postérieure, vue latérale; $\mathrm{F}$ et $\mathrm{G}$ : extrémité caudale, vues ventrale et latérale; $\mathrm{H}$ : début de l'ovéjecteur ; $\mathrm{I}$ : microfilaire utérine. $\mathrm{J}$ à $\mathrm{P}$, mâle; $\mathrm{J}$ : région caudale, vue latérale ; $\mathrm{K}$ et $\mathrm{L}$ : extrémité caudale, vues ventrale et latérale; $\mathrm{M}$ : aspect de l'area rugosa, vue ventrale ; $\mathrm{N}$ et $\mathrm{O}$ : spicule droit en vues latérale et ventrale, après dissection; P : spicule gauche, vue latérale, après dissection (apex du manche brisé). $\mathrm{Q}$ à $\mathrm{U}, D$. (A.) viteae. $\mathrm{Q}$ : tête, vue apicale; $\mathrm{R}$ et $\mathrm{S}$ : extrémité caudale d'une femelle, vues ventrale et latérale; $\mathrm{T}$ et $\mathrm{U}$ : un spicule droit disséqué, vues latérale et ventrale. (A: éch. $500 \mu$; B : éch. $100 \mu ; \mathrm{C}, \mathrm{D}, \mathrm{F}, \mathrm{G}, \mathrm{I}, \mathrm{K}$ à $\mathrm{U}$ : éch. $50 \mu ; \mathrm{E}$ : éch. $200 \mu ; \mathrm{H}$ : éch. $150 \mu$ ). 


\section{Développement larvaire}

\section{Microfilaire (en coloration vitale) (fig. 2).}

Le corps est long de 270 à $318 \mu$ et large de 5 à $5,5 \mu$. La microfilaire est à peine amincie dans la région antérieure; la tête est ronde et porte un crochet puissant - comprenant une base large et longue et une pointe terminale - et, sur la face opposée, une petite pointe cuticulaire. L'emplacement du futur anneau nerveux est marqué par 2 petites cellules nerveuses initiales. La cellule excrétrice est mince et allongée. Le corps interne est composé de 2 ou 3 masses réfringentes. La cellule $R_{1}$ est nettement séparée du groupe $R_{2}-R_{4}$. La queue est longue, effilée, à pointe mousse ou aiguë.

Pour une microfilaire longue de $270 \mu$ et large de $5 \mu$, anneau nerveux, pore excréteur et noyau excréteur à $49 \mu, 85 \mu$ et $98 \mu$ de l'apex. Crochet long de $4 \mu$. Corps interne long de $17 \mu . R_{1}, R_{2}$ et $R_{4}$ à $185 \mu, 196 \mu$ et $202 \mu$ de l'apex. Queue longue de $66 \mu$.

\section{Larves de 14 jours.}

Une dizaine de larves ont été récoltées chez un Ornithodore disséqué 14 jours après le gorgement. Elles sont longues de 600 à $700 \mu$ et correspondent à des stades II âgés. L'exuvie du stade I est très décollée dans la région antérieure; la queue se tremine par une pointe obtuse simple à l'intérieur de laquelle se dessinent déjà chez certaines larves les pointes du stade infestant; de plus, le long filament caudal de l'exuvie du premier stade est bien visible. La capsule buccale est courte et faiblement cuticularisée; l'œsophage est divisé en portions musculaire et glandulaire, sensiblement de même longueur. La cellule excrétrice est ronde et volumineuse. L'intestin est plus long que l'œsophage; le rectum est volumineux et allongé, fermé par le bouchon cuticulaire très saillant.

Pour une larve longue de $600 \mu$ et large de $28 \mu$, capsule buccale haute de $4 \mu$, anneau nerveux et cellule excrétrice à 66 et $92 \mu$ de l'apex; œsophage long de $195 \mu$ (dont $105 \mu$ de portion glandulaire); intestin, rectum et queue longs de $310 \mu$, $33 \mu$ et $50 \mu$.

\section{Forme infestante.}

Six Ornithodores ont été disséqués 24 jours après le gorgement; l'un d'entre eux contenait 2 stades infestants.

Celui qui est décrit est une larve femelle qui mesure $1585 \mu$ de long et $21 \mu$ de large. La capsule buccale est haute de $4 \mu$. La tête porte 8 papilles, en 2 cercles de 4 papilles. L'œsophage comprend une portion musculaire de $195 \mu$ et une portion glandulaire de $660 \mu$. L'anneau nerveux est à mi-hauteur de l'œsophage musculaire, à $100 \mu$ de l'apex. Le rectum est long de $65 \mu$. La queue est longue de 

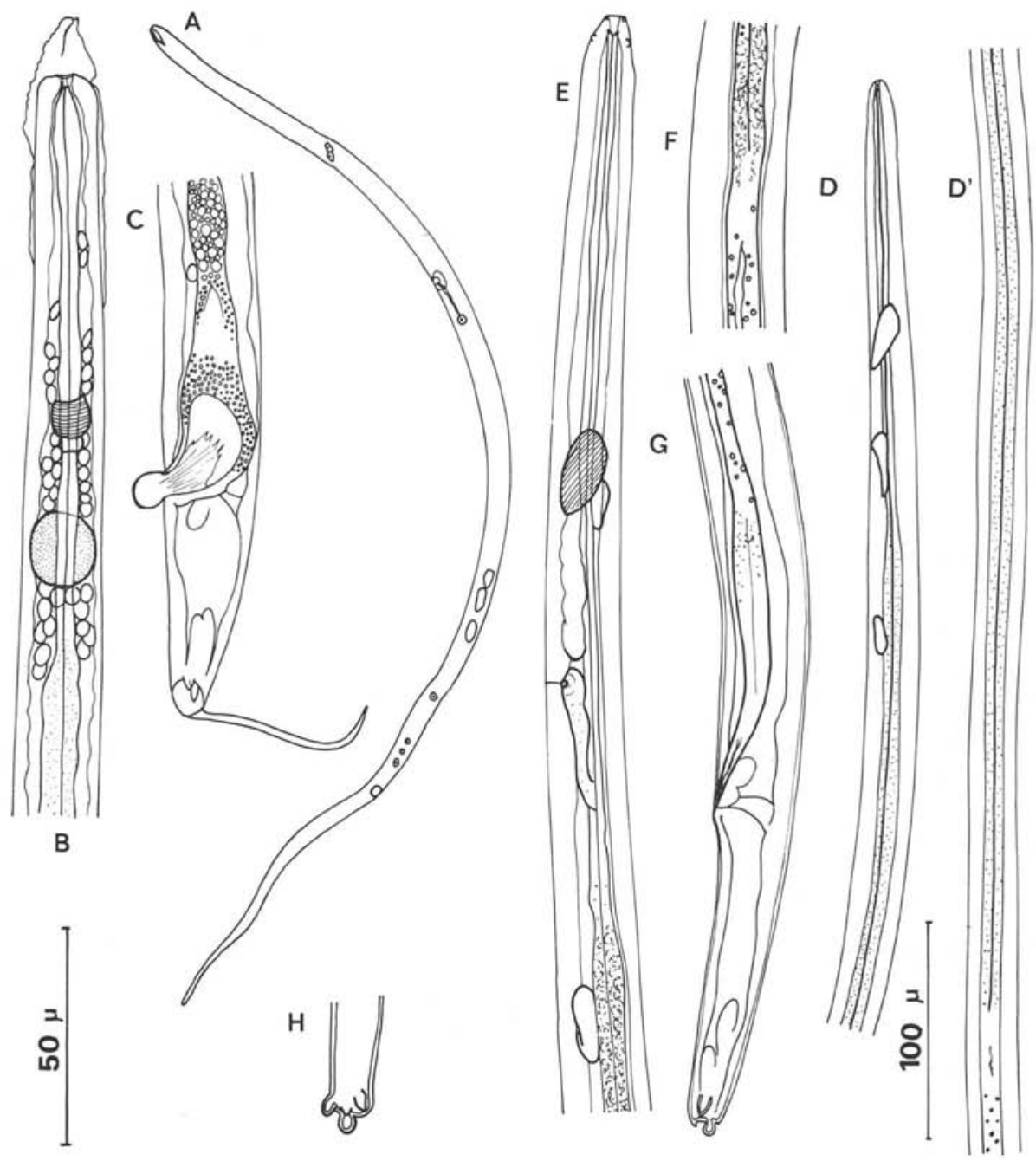

FIG. 2. - D. (A.) weissi. A : microfilaire sanguine en coloration vitale; B et C: région antérieure (vue dorsale) et postérieure (vue latérale) d'un stade II de 14 jours; D à $\mathrm{H}$, forme infestante femelle; $\mathrm{D}$ et $\mathrm{D}^{\prime}$ : région œsophagienne, vue latérale; $\mathrm{E}$; région antérieure, vue latérale ; F : jonction œsophage-intestin ; G : région caudale, vue latérale ; $\mathrm{H}$ : extrémité caudale, vue ventrale. (A, B, C, E, F, G, H : éch. $50 \mu$; D, D' : éch. $100 \mu$ ). 
$65 \mu$. et terminée de façon complexe: l'extrémité caudale est arrondie, en position subterminale se trouvent 2 languettes latéro-ventrales pointues; il existe en plus une petite pointe ventrale et une petite pointe dorsale. L'ébauche génitale est située près du début de l'œsophage glandulaire à $225 \mu$ de l'apex.

\section{Conclusion}

1. Caractères distinctifs entre les adultes de $D$. (A.) weissi et $D$. (A.) viteae.

Chabaud (1952), en décrivant $D$. (A.) viteae $(=D$. blanci), indique que le caractère qui distingue cette espèce de $D$. (A.) weissi est la longueur de l'œsophage ; chez D. (A.) viteae, le corps est long ( $\delta^{*}: 3,8$ et $4,1 \mathrm{~cm} ; q: 4,9$ et $7,2 \mathrm{~cm}$ ) et l'œsophage mesure $1600 \mu$; chez $D$. (A.) weissi, le corps est beaucoup plus court $\left(\delta^{*}: 1,5\right.$ à $1,7 \mathrm{~cm} ; \uparrow: 2,9 \mathrm{~cm}$ ) et l'œsophage mesure $2100 \mu$ chez le mâle et $3600 \mu$ chez la femelle.

La valeur de ce caractère distinctif est confirmée: les mensurations effectuées sur notre matériel sont analogues à celles de Seurat; un lot de $D$. (A.) viteae (souche entretenue sur Hamster) donne les mesures suivantes: $2 q$ longues de 4,9 et $4,7 \mathrm{~cm}$ avec des œsophages longs de 1550 et $1660 \mu ; 2 \delta$ longs de 3,28 et $3,25 \mathrm{~cm}$ et des œsophages longs de 1900 et $1600 \mu$.

Les nouvelles données morphologiques sur D. (A.) weissi n'ont pas apporté d'autres caractères distinctifs: les papilles dorso-latérales. l'area rugosa en rangées transversales de bâtonnets, les rides du spicule droit, le crochet céphalique de la microfilaire sont analogues chez les 2 espèces; chez $D$. (A.) weissi comme chez $D$. (A.) viteae, les papilles caudales sur la région moyenne de la queue sont présentes ou absentes selon les spécimens observés; les extrémités caudales des femelles des 2 espèces, nettement différentes sur certains spécimens, sont identiques sur d'autres.

\section{Analogie de la biologie larvaire de $D$. (A.) weissi et $D$. (A.) viteae.}

Le cycle de $D$. (A.) weissi a été obtenu au laboratoire chez $O$. erraticus (souche Vellozo). Sur 7 Ornithodores disséqués, qui étaient gorgés, 2 sont positifs, l'un avec une dizaine de larves en fin de stade II, l'autre avec 2 larves infestantes obtenues le $24^{\circ}$ jour à $27^{\circ} \mathrm{C}$.

La biologie de $D$. (A.) weissi, révélée par ce cycle expérimental, est tout à fait analogue à celle de $D$. (A.) viteae, dont le développement s'effectue très bien chez O. tartakowskyi et plus ou moins bien chez quelques autres Ornithodores (Bain, 1967).

Aucun caractère distinctif entre les stades infestants des 2 espèces n'a pu être mis en évidence: les longueurs de l'œsophage par rapport au corps sont semblables [0,53 chez D. (A.) weissi; 0,51 chez $D$. (A.) viteae $]$; les différences de la position de l'anneau nerveux et du pore excréteur (à 100 et $150 \mu$ de l'apex chez viteae selon Chabaud, 1954) ne sont pas confirmées par de nouvelles mesures sur D. (A.) viteae 
(pour 2 larves longues de $1750 \mu$ et $1675 \mu$, l'anneau nerveux est à 115 et $105 \mu$ de l'apex, le pore excréteur à 170 et $170 \mu$ de l'apex).

L'hypothèse selon laquelle $D$. (A.) viteae, parasite de Rongeurs Gerbillidae, avec des caractères morphologiques remarquablement primitifs (capsule buccale développée, distinction entre œsophage musculaire et glandulaire très nette, etc...), provient d'une capture de Dipetalonema d'Insectivores (Chabaud et Bain, 1976), est donc étayée par les nouvelles données sur la biologie larvaire de $D$. (A.) weissi, parasite de Macroscélide.

\section{Bibliographie}

BAIN (O.), 1967. - Biologie larvaire et mécanisme de transmission de la Filaire Dipetalonema viteae. Ann. Parasitol. hum. comp., 42, 211-267.

BAIN (O.), 1972. - Recherches sur la morphogenèse des Filaires chez l'hôte intermédiaire. Ann. Parasitol. hum. comp., 47, 251-303.

Balthazard (M.), Chabaud (A.-G.) et Minou (A.), 1952. - Cycle évolutif d'une filaire parasite de Mćrion. C.R. Acad. Sci., 234, 2115-2117.

Chabaud (A.-G.), 1952. - Le genre Dipetalonema Diesing, 1861; essai de classification. Ann. Parasitol. hum. comp., 27, 250-285.

Chabaud (A.-G.), 1954. - Sur le cycle évolutif des Spirurides et des Nématodes ayant une biologie comparable. Valeur systématique des caractères biologiques. Ann. Parasitol. hum. comp., 29, 42-88, 206-249, 358-425.

Chabaud (A.-G.) et Bain (O.), 1976. - La lignée Dipetalonema. Nouvel essai de classification. Ann. Parasitol. hum. comp., 51, 365-397.

Seurat (L.-G.), 1914. - Sur une Filaire péritonéale du Macroscélide. C.R. Si. Soc. Biol., $77,524-526$. 